\title{
Was the diagnosis really postural orthostatic tachycardia syndrome?
}

\section{Letter to the Editor}

Cite this article: Bourne KM and Raj SR (2020) Was the diagnosis really postural orthostatic tachycardia syndrome? Cardiology in the Young 30: 1978-1979. doi: 10.1017/

S1047951120003959

Received: 16 October 2020

Accepted: 18 October 2020

First published online: 16 November 2020

\section{Author for correspondence:}

Kate M. Bourne, BSc, University of Calgary, 3280 Hospital Dr NW Calgary, c/o HRIC GAC82, Calgary, AB T2N 4Z6, Canada.

Tel: +1 (403) 210-7401; Fax: +1 (403) 210-9444.

E-mail: kate.bourne1@ucalgary.ca
๑) The Author(s), 2020. Published by Cambridge University Press.

\section{CAMBRIDGE} UNIVERSITY PRESS
Kate M. Bourne (i) and Satish R. Raj (i)

Department of Cardiac Sciences, Cumming School of Medicine, University of Calgary, Calgary, AB, Canada

We were interested in the recent report entitled "A Pilot Study using the Compensatory Reserve Index to evaluate individuals with Postural Orthostatic Tachycardia Syndrome". Unfortunately, the authors did not adequately test this hypothesis.

The main challenge is that they did not study patients with Postural Orthostatic Tachycardia Syndrome. The authors correctly point out that currently accepted criteria for a diagnosis of Postural Orthostatic Tachycardia Syndrome in adolescents (12-19 years of age) include a heart rate increase of 40 beats/minute or more within 10 minutes of upright posture, in the absence of orthostatic hypotension $(20 / 10 \mathrm{mmHg}$ decrease $) .{ }^{1-3}$ Recent expert consensus statements confirm these diagnostic criteria. ${ }^{1-3}$ Shahi et al chose to disregard this criterion in their study and elected to use a 30 beats/minute heart rate increase, based on a musing from a single paper.

Unfortunately, only 8 of 44 putative Postural Orthostatic Tachycardia Syndrome patients met even this lower bar (and at most 2 patients met the conventional heart rate criterion). We do not understand how the authors can report on a study evaluating patients with Postural Orthostatic Tachycardia Syndrome, when they really did not have patients with Postural Orthostatic Tachycardia Syndrome enrolled in the study. It is possible that the Compensatory Reserve Index will be a valuable tool in understanding Postural Orthostatic Tachycardia Syndrome physiology, but this study does not help in that determination.

Although not addressed in the article title or the stated goals of the paper, the authors' efforts do point out potential problems in the diagnosis of Postural Orthostatic Tachycardia Syndrome. Clearly, the vast majority of their participants with a pre-existing diagnosis of Postural Orthostatic Tachycardia Syndrome do not seem to meet the criteria. This could be for a variety of reasons. First, it is possible that these patients did have Postural Orthostatic Tachycardia Syndrome, but that they have undergone exceptional treatment. Some patients can improve with dietary salt loading and aerobic exercise reconditioning.

The second possibility is that patients were on medications during the study that blunted their orthostatic tachycardia. The investigators excluded patients taking beta-blockers (not just asking this to be held), and very few patients were taking other drugs commonly used to treat Postural Orthostatic Tachycardia Syndrome (midodrine and fludrocortisone).

The third possibility is that these patients were not diagnosed with Postural Orthostatic Tachycardia Syndrome by a physician. Getting a diagnoses with chronic illness can be difficult and frustrating for patients, and many patients will self-diagnose.

A final, and more concerning, possibility is that these patients were incorrectly diagnosed with Postural Orthostatic Tachycardia Syndrome by their physicians. As awareness of Postural Orthostatic Tachycardia Syndrome increases in the physician community, so does the potential for misdiagnosis. If this latter possibility is the problem, then this is an important finding that points to a significant knowledge gap that needs to be addressed. Review of medical records would be required to discriminate between the latter two possibilities, but it is not clear if this was done.

In summary, the authors might be reporting an important finding (about the inconsistency of a Postural Orthostatic Tachycardia Syndrome diagnosis), but that finding is not about whether or not the Compensatory Reserve Index is a useful tool in Postural Orthostatic Tachycardia Syndrome patients. To test that hypothesis, they would need to study patients with Postural Orthostatic Tachycardia Syndrome.

Acknowledgements. The authors would like to acknowledge the patients who volunteer their time to participate in our research program.

Financial support. This work received no specific grant from any funding agency, commercial or not-for-profit sectors.

Conflicts of interest. K.B.: none; S.R.R.: Consultant for Lund beck NA Ltd and Theravance Biopharma, Chair, Data Safety and monitoring Board for Arena Pharmaceuticals; Cardiac Arrhythmia Network of Canada (CANet; London, ON, Canada) Network Investigator; Medical Advisory Board of Dysautonomia International and POTS UK, both without financial compensation. 


\section{References}

1. Raj SR, Guzman JC, Harvey P, et al. Canadian cardiovascular society position statement on postural orthostatic tachycardia syndrome (POTS) and related disorders of chronic orthostatic intolerance. Can J Cardiol 2020; 36: 357-372.

2. Sheldon RS, Grubb BP, Olshansky B, et al. 2015 heart rhythm society expert consensus statement on the diagnosis and treatment of postural tachycardia syndrome, inappropriate sinus tachycardia, and vasovagal syncope. Heart Rhythm 2015; 12: e41-e63.

3. Freeman R, Wieling W, Axelrod FB, et al. Consensus statement on the definition of orthostatic hypotension, neutrally mediated syncope and the postural tachycardia syndrome. Clin Auton Res 2011; 21: 69-72. 